\title{
EDITORIAL
}

\section{The hope of progress}

\author{
Miranda Robertson*
}

"The Hope of Progress" is the title of a collection of essays [1] by Peter Medawar, who won the Nobel Prize in Physiology or Medicine in 1960, jointly with Frank McFarlane Burnet, for his research in immunology. The sentence from which the phrase is taken - "To deride the hope of progress is the ultimate fatuity, the last word in poverty of spirit and meanness of mind" - may strike us as overelaborate and flowery in this texting and blogging age when the analogous sentiment is expressed as "Yes we can". But Medawar was remarkable for his clarity of thought, as well as for his (now unfashionable) elegance of expression, and his research on transplant rejection led to the discovery of principles fundamental to modern immunology; so the phrase seems apt as a title for a new series that we are launching with three contributions on biology relevant to clinical problems.

Two of the three contributions are reviews - by Christopher Lord and Alan Ashworth on the development of new cancer therapeutic drugs [2], and by Amy McKee, Megan McLeod, John Kappler and Philippa Marrack [3] on adjuvants and vaccine development. The third is a new feature, a video Q\&A (see [4]), in which Martin Raff explains his interest in, and delivers his views on research on the biological basis of autism, both in video and in text format [5].

\section{The rationalization of cancer therapy}

Alan Ashworth and Christopher Lord are known for the ingenious application of poly(ADP-ribose) inhibitors to the treatment of $B R C A$-mutant tumors, an approach that migrated from laboratory bench to phase II clinical trials in less than five years. This work, an application of the synthetic lethal principle borrowed from genetic analysis and applied to tumor therapy, is described in their review, which traces the evolution of anti-cancer drugs from the cytotoxic blunt instruments that remain the principal weapons against cancer to date, through the more refined and sophisticated drugs - notably herceptin and imatinib - targeted at molecules known to be modified in specific tumors, and finally describes how an understanding of

*Correspondence: bmcbiologyeditorial@biomedcentral.com the workings of cells as a whole, and the entire panoply of changes that characterize a tumor cell, and not just a single mutant molecule, will become the basis of drug treatment.

\section{The design of vaccines}

Vaccination, the first great empirical success of immunology, has, by comparison with the major chemotherapeutic drugs, the properties of a magic bullet. It is a remarkable fact, therefore, that despite an increasingly detailed appreciation of the workings of the immune system, the effectiveness of vaccines is still not properly understood. We understand that vaccination generates an adaptive immune response, usually protective antibodies; but this is the end result of a process of several cell-cell interactions that determine, first, whether there is an immune response at all; and second, whether that response is protective for a given pathogen. The induction of these protective immune responses, as well as how they are amplified and tuned (as opposed to targeted), depends upon adjuvants, which can be substances added to a vaccine, or can be properties inherent in an intact pathogen.

Tantalizingly, while some of the cellular responses and some of the cell-cell interactions underlying adjuvant effects are understood in principle, this understanding falls crucially short of allowing the design of vaccines to produce lasting immunity of the appropriate kind to all the infectious diseases flesh is heir to. Some vaccines induce reliable immunity that lasts for many years, while for some diseases there is no effective vaccine at all.

In their review, McKee et al. [3] discuss what is known of adjuvant effects, what remains to be discovered, and where the most promising paths to progress lie.

\section{Research and reality}

While research on the biological basis of vaccination and targets for tumor therapy can justly be called translational, it would be fairer to describe the genomics of psychiatric disease as pre-translational. This contentious field is touched on by Martin Raff, in the interview we recorded with him and which, with the edited transcript, is the third of the first three publications in the Hope of progress series. Alan Ashworth believes strongly in the critical importance of understanding the clinical reality 
of the cancers whose conquest is the aim of research biologists, and thus the need to foster close collaboration between clinicians and laboratory scientists [2, 6]; and it is notable how little much of the research on the molecular and cell biology underlying clearly clinical problems seems to reflect an awareness of the broader picture of the disease. In the third of our three Hope of progress articles, on autism, Martin Raff explicitly embraces all levels of description, from the behaviour of the autistic child to the single nucleotides upon whose polymorphisms current notions of the underlying genetic architecture are built. He can best speak for himself [5] on how the autistic phenotype(s) might be reconciled with a genetically determined neurodevelopmental problem - autism being among the most heritable of the psychiatric disorders (and he would be the first to point out that he speaks on this as an interested - in both senses of the word -nonpractitioner). But the issues that he raises about the pursuit of that heritability are general to all heritable psychiatric disorders - and indeed to genetically complex non-psychiatric disorders as well.

\section{The genetic architecture of psychiatric disease}

It has long been known on the basis of family studies and studies of twins and adopted children that there is a heritable component in psychiatric disease, and given that psychiatric disease is common - schizophrenia for example affects up to $0.8 \%$ of the population - but does not seem to follow a Mendelian pattern of inheritance, a widely embraced notion has been that it is caused by the combined effects of a large number of common genetic variants each with an individually small effect. This is known as the common disease-common variant ( $\mathrm{CDCV}$ ) hypothesis. The alternative proposition is that these diseases are caused by rare variants with large effects, and they are common because there are many such rare variants, which arise with relatively high frequency de novo. The case for rare variants with large effects has been persuasively argued for schizophrenia by McClellan et al. [7] and by Mitchell and Porteous [8], and for complex traits in general by Goldstein [9]. One of the main planks in the argument against the common disease-common variant hypothesis is that in genomewide association studies (GWAS) only a tiny percentage of the heritability can be explained by common singlenucleotide polymorphisms (SNPs) [10], an embarrassment that is discussed by John Brookfield in a Q\&A article [11] published to accompany our Hope of Progress articles and in which he explains the population genetic issues that arise in the interpretation of association studies. This makes the alternative hypothesis of many, highly penetrative, rare mutations in many different genes attractive; and several such variants with strong effects have been found.
The two proposed mechanisms of heritability are not mutually exclusive however, and there are many possibilities: Mitchell and Porteous [8] suggest, for example, that common low-penetrance variants may be important modifiers of the effects of rare high-penetrance ones. The contention arises because, first, the basis for recent claims to have identified common variants underlying psychiatric disease is susceptible to challenge [8], and second, the two mechanisms have different implications for research strategy. Under the common disease-common variant hypothesis, the appropriate strategy is to increase the sample sizes for the genomes scanned in association studies, for reasons explained by Brookfield in his Q\&A article [11]. If on the other hand rare de novo variants of large effect are important, then it will be more fruitful to identify individual families in which such mutant genes may be identified, and genomewide scans of large populations are unlikely to be helpful because different genes are likely to be affected in different families. (This will not necessarily mean however that different families will have distinct disease phenotypes: first, different affected genes may be on the same pathway; and second, one of the most astonishing revelations in those cases in which a variant gene of large effect has been identified is that the disease phenotype can vary enormously within a family, embracing diagnoses as different as bipolar and schizophrenic psychoses.)

\section{What next?}

Eventually, it is reasonable to suppose, by one method or the other, variant genes at the root of these disorders will be identified and assigned to pathways, and the relative roles of highly penetrant and small-effect variants will become clear. We shall then be left with a deeper mystery: why is the concordance rate for schizophrenia in monozygotic - and therefore genetically identical twins, even if they are reared together, only about $50 \%$ ? The non-genetic causes of psychiatric disease may perhaps be a question for the biology of systems.

Future articles in the Hope of progress series will address what genomics can offer to the study of the response to infectious disease, the problems of vaccinating children in undeveloped countries, and protein folding problems underlying neurological disease. The answer to the monozygotic twin mystery may have to wait for another series.

Published: 12 April 2010

\section{References}

1. Medawar PB: The hope of progress 1972, Methuen, London

2. Lord CJ, Ashworth A: Biology driven cancer drug development: back to the future. BMC Biology 2010, 8:38

3. McKee AS, MacLeod MKL, Kappler JW, Marrack P: Immune mechanisms of protection: can adjuvants rise to the challenge? BMC Biology 2010, 8:37

4. Robertson M: Plus ça change. BMC Biology 2010, 8:44 
5. Raff M: Video Q\&A: What is autism?-A personal view. BMC Biology 2010, 8:42

6. Cressey D: Talking up translation. Nature 2010, 463:422-423.

7. McClellan JM, Susser E, King M-C: Schizophrenia: a common disease caused by multiple rare alleles. Brit J Psychiatry 2007, 190:194-199.

8. Mitchell KJ, Porteous DJ: Rethinking the genetic architecture of schizophrenia. Psychol Med 2010 (in the press)

9. Goldstein DB: Common genetic variation and human traits N Engl J Med 2009, 360:1696-1698.

10. Manolio TA, Collins FS, Cox NJ, Goldstein DB, Hindorff LA, Hunter DJ, McCarthy MI, Ramos EM, Cardon LR, Chakravarti A, Cho JH, Guttmacher AE, Kong A, Kruglyak L, Mardis E, Rotimi CN, Slatkin M, Valle D, Whittemore AS, Boehnke M, Clark AG, Eichler EE, Gibson G, Haines JL, Mackay TF, McCarroll SA,
Visscher PM: Finding the missing heritability of complex diseases. Nature 2009, 461:747-753.

11. Brookfield JFY: Promise and pitfalls of genome-wide association studies. BMC Biology 2010, 8:41

doi:10.1186/1741-7007-8-39

Cite this article as: Robertson M: The hope of progress. BMC Biology 2010 8:39. 